\title{
An Analytical Solution in the Complex Plane for the Luminosity Distance in Flat Cosmology
}

\author{
Lorenzo Zaninetti \\ Physics Department, Via P. Giuria 1, Turin, Italy \\ Email: zaninetti@ph.unito.it
}

How to cite this paper: Zaninetti, L. (2016) An Analytical Solution in the Complex Plane for the Luminosity Distance in Flat Cosmology. Journal of High Energy Physics, Gravitation and Cosmology, 2, 581-586. http://dx.doi.org/10.4236/jhepgc.2016.24050

Received: June 27, 2016

Accepted: September 10, 2016

Published: September 13, 2016

Copyright $\odot 2016$ by author and Scientific Research Publishing Inc. This work is licensed under the Creative Commons Attribution International License (CC BY 4.0).

http://creativecommons.org/licenses/by/4.0/

\begin{abstract}
We present an analytical solution for the luminosity distance in spatially flat cosmology with pressureless matter and the cosmological constant. The complex analytical solution is made of a real part and a negligible imaginary part. The real part of the luminosity distance allows finding the two parameters $H_{0}$ and $\Omega_{\mathrm{M}}$. A simple expression for the distance modulus for SNs of type Ia is reported in the framework of the minimax approximation.
\end{abstract}

\section{Keywords}

Cosmology, Observational Cosmology, Distances, Redshifts, Radial Velocities, Spatial Distribution of Galaxies

\section{Introduction}

The luminosity distance in flat cosmology has been recently investigated using different approaches. A fitting formula which has a maximum relative error of $4 \%$ in the case of common cosmological parameters has been introduced by [1]. An approximate solution in terms of Padé approximants has been presented by [2]. The integral of the luminosity distance has been found in terms of elliptical integrals of the first kind by [3].

\section{Flat Cosmology}

Following Equation (2.1) in [2], the luminosity distance $d_{\mathrm{L}}$ is

$$
d_{\mathrm{L}}\left(z ; c, H_{0}, \Omega_{\mathrm{M}}\right)=\frac{c}{H_{0}}(1+z) \int_{\frac{1}{1+z}}^{1} \frac{\mathrm{d} a}{\sqrt{\Omega_{\mathrm{M}} a+\left(1-\Omega_{\mathrm{M}}\right) a^{4}}},
$$

where $H_{0}$ is the Hubble constant expressed in $\mathrm{km} \cdot \mathrm{s}^{-1} \cdot \mathrm{Mpc}^{-1}, c$ is the speed of light expressed in $\mathrm{km} \cdot \mathrm{s}^{-1}, z$ is the redshift, $a$ is the scale-factor, and $\Omega_{\mathrm{M}}$ is 


$$
\Omega_{\mathrm{M}}=\frac{8 \pi G \rho_{0}}{3 H_{0}^{2}},
$$

where $G$ is the Newtonian gravitational constant and $\rho_{0}$ is the mass density at the present time. We now introduce the indefinite integral

$$
\Phi(a)=\int \frac{\mathrm{d} a}{\sqrt{\Omega_{\mathrm{M}} a+\left(1-\Omega_{\mathrm{M}}\right) a^{4}}} .
$$

The solution is in terms of $F$, the Legendre integral or incomplete elliptic integral of the first kind

$$
\Phi(a)=\frac{-4 F\left(b_{1}, b_{2}\right) b_{3} b_{4} b_{6} b_{1} b_{5}}{b_{7} b_{8} \sqrt{b_{9}} b_{10}},
$$

where the incomplete elliptic integral of the first kind is

$$
F(x, k)=\int_{0}^{x} \frac{\mathrm{d} t}{\sqrt{1-t^{2}} \sqrt{1-k^{2} t^{2}}},
$$

see formula (19.2.4) in [4], and

$$
\begin{aligned}
& b_{1}=\sqrt{-\frac{a\left(\Omega_{\mathrm{M}}-1\right)(i \sqrt{3}+3)}{\left(-\Omega_{\mathrm{M}} a+\sqrt[3]{\Omega_{\mathrm{M}}\left(\Omega_{\mathrm{M}}-1\right)^{2}}+a\right)(i \sqrt{3}+1)},} \\
& b_{2}=\sqrt{\frac{(i \sqrt{3}+1)(i \sqrt{3}-3)}{(i \sqrt{3}+3)(i \sqrt{3}-1)}}, \\
& b_{3}=\sqrt{\frac{i \sqrt{3} \sqrt[3]{\Omega_{\mathrm{M}}\left(\Omega_{\mathrm{M}}-1\right)^{2}}+2 \Omega_{\mathrm{M}} a+\sqrt[3]{\Omega_{\mathrm{M}}\left(\Omega_{\mathrm{M}}-1\right)^{2}}-2 a}{\left(-\Omega_{\mathrm{M}} a+\sqrt[3]{\Omega_{\mathrm{M}}\left(\Omega_{\mathrm{M}}-1\right)^{2}}+a\right)(i \sqrt{3}+1)},} \\
& b_{4}=\sqrt{\frac{-i \sqrt{3} \sqrt[3]{\Omega_{\mathrm{M}}\left(\Omega_{\mathrm{M}}-1\right)^{2}}+2 \Omega_{\mathrm{M}} a+\sqrt[3]{\Omega_{\mathrm{M}}\left(\Omega_{\mathrm{M}}-1\right)^{2}}-2 a}{\left(-\sqrt[3]{\Omega_{\mathrm{M}}\left(\Omega_{\mathrm{M}}-1\right)^{2}}+a\left(\Omega_{\mathrm{M}}-1\right)(i \sqrt{3}-1)\right.}}, \\
& b_{5}=i \sqrt{3}+1, \\
& b_{6}=\left(-\Omega_{\mathrm{M}} a+\sqrt[3]{\Omega_{\mathrm{M}}\left(\Omega_{\mathrm{M}}-1\right)^{2}}+a\right)^{2}, \\
& b_{7}=\sqrt[3]{\Omega_{\mathrm{M}}\left(\Omega_{\mathrm{M}}-1\right)^{2}}, \\
& b_{8}=i \sqrt{3}+3, \\
& b_{9}=\left(-4 a^{4}+4 a\right) \Omega_{\mathrm{M}}+4 a^{4}, \\
& b_{10}=\Omega_{\mathrm{M}}-1,
\end{aligned}
$$

with $i^{2}=-1$. The incomplete elliptic integral $F(x, k)$ of complex arguments is evaluated according to Equation (17.4.11) in [5] or Section 19.7 (ii) in [4]. The luminosity distance is 


$$
d_{\mathrm{L}}\left(z ; c, H_{0}, \Omega_{\mathrm{M}}\right)=\Re\left(\frac{c}{H_{0}}(1+z)\left(\Phi(1)-\Phi\left(\frac{1}{1+z}\right)\right)\right),
$$

where $\mathfrak{R}$ means the real part.

The distance modulus is

$$
(m-M)=25+5 \log _{10}\left(d_{\mathrm{L}}\left(z ; c, H_{0}, \Omega_{\mathrm{M}}\right)\right) .
$$

An approximation can be found when the argument of the integral (1) is expanded about $a=1$ in a Taylor series of order 10. The resulting Taylor approximation of order 10 to the luminosity distance, $d_{\mathrm{L}}\left(z ; c, H_{0}, \Omega_{\mathrm{M}}\right)_{10}$, is

$$
\begin{aligned}
& d_{\mathrm{L}}\left(z ; c, H_{0}, \Omega_{\mathrm{M}}\right)_{10}= \\
& \frac{c(1+z)}{H_{0}}\left(\frac{1}{2}\left(\frac{3}{2} \Omega_{\mathrm{M}}-2\right)\left(1-(1+z)^{-2}\right)+3-3(1+z)^{-1}\right. \\
& \left.-\frac{3}{2} \Omega_{\mathrm{M}}\left(1-(1+z)^{-1}\right)\right)+\cdots
\end{aligned}
$$

where we have reported the first few terms of the series. The goodness of the Taylor approximation is evaluated through the percentage error, $\delta$, which is

$$
\delta=\frac{\left|d_{\mathrm{L}}\left(z ; c, H_{0}, \Omega_{\mathrm{M}}\right)-d_{\mathrm{L}}\left(z ; c, H_{0}, \Omega_{\mathrm{M}}\right)_{10}\right|}{d_{\mathrm{L}}\left(z ; c, H_{0}, \Omega_{\mathrm{M}}\right)} \times 100 .
$$

As an example when $H_{0}=70 \mathrm{~km} \cdot \mathrm{s}^{-1} \cdot \mathrm{Mpc}^{-1}, \Omega_{\mathrm{M}}=0.3, \quad c=299792.458 \mathrm{~km} \cdot \mathrm{s}^{-1}$ and $z=4$, we obtain $\delta=0.61 \%$. As an example with the above parameters, $d_{\mathrm{L}}$ has its angle in the complex plane, $\theta$, very small: $\theta \approx 10^{-11}$, which means that the solution is real for practical purposes. In the last years the Hubble Space Telescope (HST) has allowed the determination of the cosmological parameters through the modulus of the distance for SNs of type Ia, see [6]-[10]. At the moment of writing the two unknown parameters, $H_{0}$ and $\Omega_{\mathrm{M}}$, can be derived from two catalogs for the distance modulus of SNs of type Ia: $580 \mathrm{SNe}$ in the Union 2.1 compilation, see [11] with data at http://supernova.lbl.gov/Union/, and $740 \mathrm{SNe}$ in the joint light-curve analysis (JLA), see [12] with data at http://supernovae.in2p3.fr/sdss_snls_jla/ReadMe.html. This kind of analysis is not new and has been used, for example, by [13].

The best fit for the distance modulus of SNs is obtained adopting the LevenbergMarquardt method (subroutine MRQMIN in [14]). The statistical parameters here adopted are the merit function or chi-square, $\chi^{2}$, the reduced chi-square, $\chi_{\text {red }}^{2}$ and the maximum probability of obtaining a better fitting, Q, see Section 2.3 in [15] for more details. Table 1 reports $H_{0}$ and $\Omega_{\mathrm{M}}$ for the two catalogs of SNs and Figure 1 and Figure 2 display the best fits.

The Taylor approximation of order 10 to the distance modulus, $d_{\mathrm{L}}\left(z ; c, H_{0}, \Omega_{\mathrm{M}}\right)_{10}$, is

$$
(m-M)_{10}=25+5 \log _{10}\left(d_{\mathrm{L}}\left(z ; c, H_{0}, \Omega_{\mathrm{M}}\right)_{10}\right) .
$$


Table 1. Numerical values of $\chi^{2}, \quad \chi_{\text {red }}^{2}$ and $Q$ where $k$ stands for the number of parameters.

\begin{tabular}{ccccccc}
\hline Compilation & SNs & $k$ & Parameters & $\chi^{2}$ & $\chi_{\text {red }}^{2}$ & $Q$ \\
\hline Union 2.1 & 580 & 2 & $H_{0}=70 \pm 0.34 ; \Omega_{\mathrm{M}}=0.277 \pm 0.019$ & 562.22 & 0.972 & 0.673 \\
JLA & 740 & 2 & $H_{0}=69.83 \pm 0.31 ; \Omega_{\mathrm{M}}=0.287 \pm 0.018$ & 627.82 & 0.85 & 0.998 \\
\hline
\end{tabular}

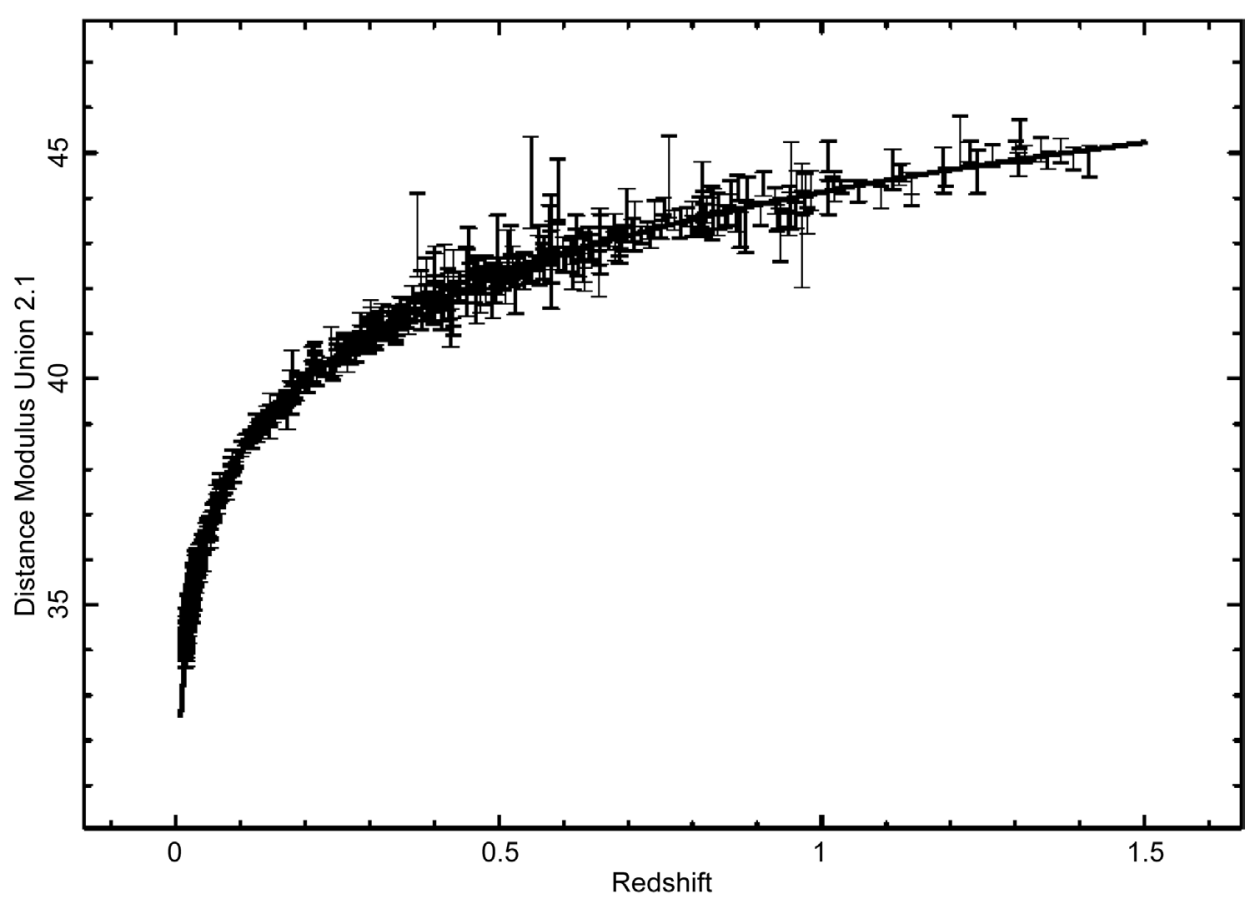

Figure 1. Hubble diagram for the Union 2.1 compilation. The solid line represents the best fit for the exact distance modulus in flat cosmology as represented by Equation (7), parameters as in first line of Table 1 .

The above equation takes a simple expression when the minimax rational approximation is used, see [4] [16] [17]; here we have used a polynomial of degree 3 for the numerator and degree 2 for the denominator. With the parameters of Table 1 for the Union 2.1 compilation over the range in $z \in[0,4]$, we obtain the following minimax approximation

$$
(m-M)_{3,2,10}=\frac{0.413991+6.080622 z+5.501967 z^{2}+0.029254 z^{3}}{0.012154+0.148352 z+0.112017 z^{2}}
$$

the maximum error being 0.002956 .

\section{Conclusion}

We have presented an analytical approximation for the luminosity distance in terms of elliptical integrals with complex argument. The fit of the distance modulus of SNs of type Ia allows finding the pair $H_{0}$ and $\Omega_{\mathrm{M}}$ for the Union 2.1 and JLA compilations. 


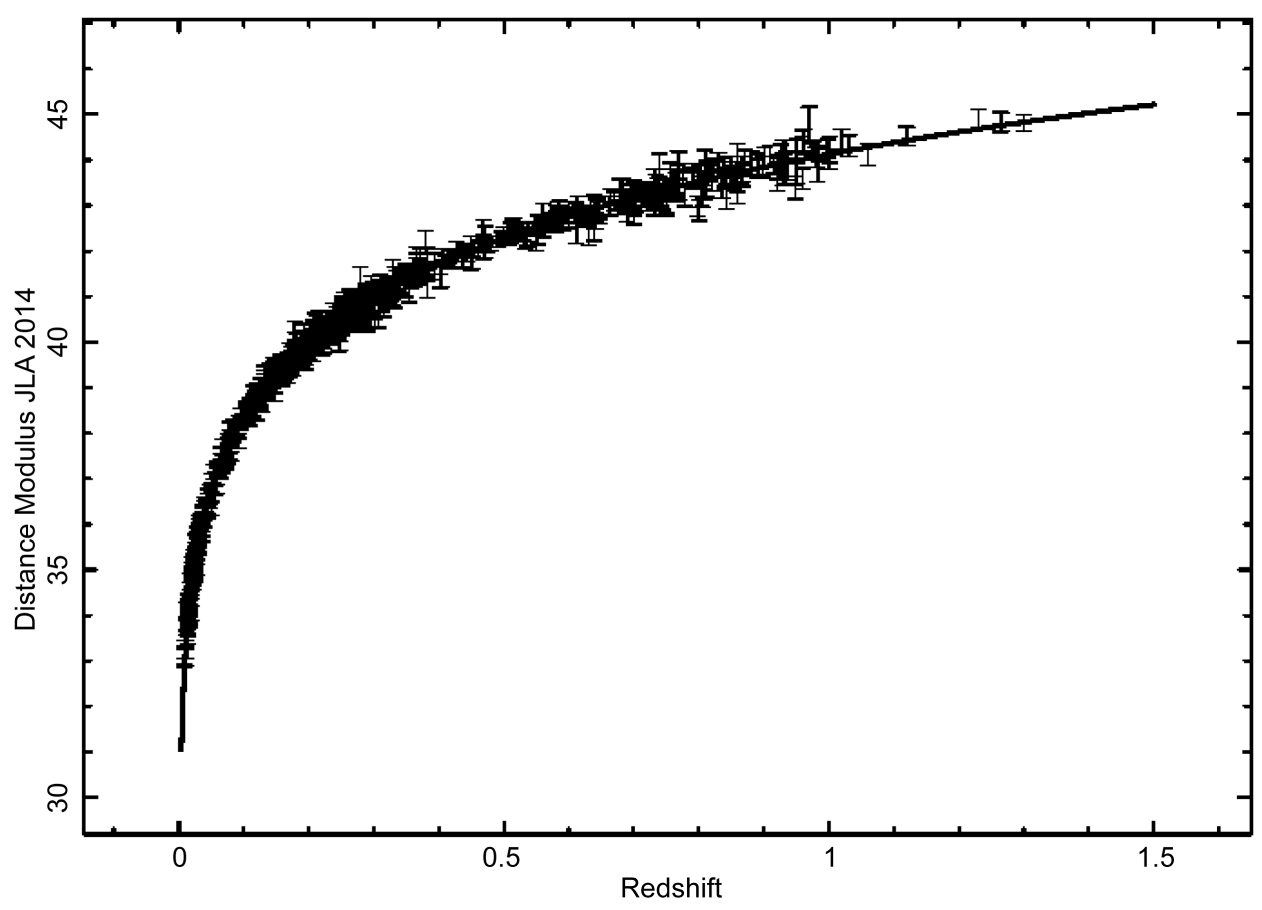

Figure 2. Hubble diagram for the JLA compilation. The solid line represents the best fit for the exact distance modulus in flat cosmology as represented by Equation (7), parameters as in second line of Table 1.

A simple expression for the distance modulus relative to the Union 2.1 compilation is given through the minimax approximation applied to a Taylor expansion of the luminosity distance of order 10 .

\section{References}

[1] Pen, U.L. (1999) Analytical Fit to the Luminosity Distance for Flat Cosmologies with a Cosmological Constant. Astrophysical Journal Supplement Series, 120, 49. http://dx.doi.org/10.1086/313167

[2] Adachi, M. and Kasai, M. (2012) An Analytical Approximation of the Luminosity Distance in Flat Cosmologies with a Cosmological Constant. Progress of Theoretical Physics, 127, 145. http://dx.doi.org/10.1143/PTP.127.145

[3] Mészáros, A. and Řpa, J. (2013) A Curious Relation between the Flat Cosmological Model and the Elliptic Integral of the First Kind. Astronomy \& Astrophysics, 556, A13. http://dx.doi.org/10.1051/0004-6361/201322088

[4] Olver, F.W.J., Lozier, D.W., Boisvert, R.F. and Clark, C.W. (2010) NIST Handbook of Mathematical Functions. Cambridge University Press, Cambridge.

[5] Abramowitz, M. and Stegun, I.A. (1965) Handbook of Mathematical Functions with Formulas, Graphs, and Mathematical Tables. Dover, New York.

[6] Perlmutter, S., Aldering, G., della Valle, M., Deustua, S., Ellis, R.S., Fabbro, S., Fruchter, A., Goldhaber, G., Groom, D.E., Hook, I.M., Kim, A.G., Kim, M.Y., Knop, R.A., Lidman, C., McMahon, R.G., Nugent, P., Pain, R., Panagia, N., Pennypacker, C.R., Ruiz-Lapuente, P., Schaefer, B. and Walton, N. (1998) Discovery of a Supernova Explosion at Half the Age of the Universe. Nature, 391, 51. http://dx.doi.org/10.1038/34124 
[7] Garnavich, P.M., Kirshner, R.P., Challis, P., Tonry, J., Gilliland, R.L., Smith, R.C., Clocchiatti, A., Diercks, A., Filippenko, A.V., Hamuy, M., Hogan, C.J., Leibundgut, B., Phillips, M.M., Reiss, D., Riess, A.G., Schmidt, B.P., Schommer, R.A., Spyromilio, J., Stubbs, C., Suntzeff, N.B. and Wells, L. (1998) Constraints on Cosmological Models from Hubble Space Telescope Observations of High-z Supernovae. Astrophysical Journal Letters, 493, L53. http://dx.doi.org/10.1086/311140

[8] Riess, A.G., Filippenko, A.V., Challis, P. and Clocchiatti, A. (1998) Observational Evidence from Supernovae for an Accelerating Universe and a Cosmological Constant. Astronomical Journal, 116, 1009. http://dx.doi.org/10.1086/300499

[9] Knop, R.A., Aldering, G., Amanullah, R., Astier, P., Blanc, G., Burns, M.S., Conley, A., Deustua, S.E., Doi, M., Ellis, R., Fabbro, S., Folatelli, G., Fruchter, A.S., Garavini, G., Garmond, S., Garton, K., Gibbons, R., Goldhaber, G., Goobar, A., Groom, D.E., Hardin, D., Hook, I., Howell, D.A., Kim, A.G., Lee, B.C., Lidman, C., Mendez, J., Nobili, S., Nugent, P.E., Pain, R., Panagia, N., Pennypacker, C.R., Perlmutter, S., Quimby, R., Raux, J., Regnault, N., Ruiz-Lapuente, P., Sainton, G., Schaefer, B., Schahmaneche, K., Smith, E., Spadafora, A.L., Stanishev, V., Sullivan, M., Walton, N.A., Wang, L., Wood-Vasey, W.M. and Yasuda, N. (2003) New Constraints on $\Omega_{M}, \Omega_{\text {Lambda }}$, and w from an Independent Set of 11 High-Redshift Supernovae Observed with the Hubble Space Telescope. Astrophysical Journal Letters, 598, 102. http://dx.doi.org/10.1086/378560

[10] Riess, A.G., Strolger, L.G., Casertano, S., Ferguson, H.C., Mobasher, B., Gold, B., Challis, P.J., Filippenko, A.V., Jha, S., Li, W., Tonry, J., Foley, R., Kirshner, R.P., Dickinson, M., MacDonald, E., Eisenstein, D., Livio, M., Younger, J., Xu, C., Dahlén, T. and Stern, D. (2007) New Hubble Space Telescope Discoveries of Type Ia Supernovae at z Greater than 1: Narrowing Constraints on the Early Behavior of Dark Energy. Astrophysical Journal Letters, 659, 98. http://dx.doi.org/10.1086/510378

[11] Suzuki, N., Rubin, D., Lidman, C., Aldering, G., Amanullah, R., Barbary, K. and Barrientos, L.F. (2012) The Hubble Space Telescope Cluster Supernova Survey. V. Improving the DarkEnergy Constraints above z Greater than 1 and Building an Early-Type-Hosted Supernova Sample. Astrophysical Journal Letters, 746, 85. http://dx.doi.org/10.1088/0004-637X/746/1/85

[12] Betoule, M., Kessler, R., Guy, J. and Mosher, J. (2014) Improved Cosmological Constraints from a Joint Analysis of the SDSS-II and SNLS Supernova Samples. Astronomy \& Astrophysics, 568, A22. http://dx.doi.org/10.1051/0004-6361/201423413

[13] Oliveira, F.J. (2016) Cosmic Time Transformations in Cosmological Relativity. Journal of High Energy Physics, Gravitation and Cosmology, 2, 253. http://dx.doi.org/10.4236/jhepgc.2016.22022

[14] Press, W.H., Teukolsky, S.A., Vetterling, W.T. and Flannery, B.P. (1992) Numerical Recipes in Fortran. The Art of Scientific Computing. Cambridge University Press, Cambridge.

[15] Zaninetti, L. (2016) Pade Approximant and Minimax Rational Approximation in Standard Cosmology. Galaxies, 4, 4. http://www.mdpi.com/2075-4434/4/1/4

[16] Remez, E. (1934) Sur la détermination des polynômes d’approximation de degré donnée. Comm. Soc. Math. Kharkov, 10, 41.

[17] Remez, E. (1957) General Computation Methods of Chebyshev Approximation. The Problems with Linear Real Parameters. Publishing House of the Academy of Science of the Ukrainian SSR, Kiev. 
Submit or recommend next manuscript to SCIRP and we will provide best service for you:

Accepting pre-submission inquiries through Email, Facebook, LinkedIn, Twitter, etc. A wide selection of journals (inclusive of 9 subjects, more than 200 journals)

Providing 24-hour high-quality service

User-friendly online submission system

Fair and swift peer-review system

Efficient typesetting and proofreading procedure

Display of the result of downloads and visits, as well as the number of cited articles

Maximum dissemination of your research work

Submit your manuscript at: http://papersubmission.scirp.org/ 